\title{
Research on Fault Diagnosis Method of NC Machine Based on Fuzzy Synthetic Discrimination
}

\author{
Mei Tian ${ }^{1, a}$, Songyu $\mathrm{Li}^{2, \mathrm{~b}}$, Chunyan You ${ }^{3, \mathrm{c}}$ and Yanhong Sun ${ }^{4, \mathrm{~d}^{*}}$ \\ ${ }^{1,2,3,4}$ College of Mechanical Engineering, Jilin Engineering Normal University, No. 3050 Kaixuan \\ Road Kuancheng District, 130052 Changchun China \\ a32593829@qq.com, b1738761582@qq.com c2206768436@qq.com, d343175460@qq.com
}

Keywords: Numerical control machine (NC machine); Fault diagnosis; Information fusion; Model; Fuzzy synthetic discrimination

\begin{abstract}
Aiming at the difficulty of fuzzy synthetic discrimination model of NC machine, this paper proposes the primary diagnosis results which change the evaluation factors from high dimensional features to the low one based on the initial diagnosis results of classifier. It reduces the complexity of the model and constructs a single stage fuzzy synthetic discrimination model for fault diagnosis of NC machine based on information fusion successfully. It puts forward the method of evaluating classifier classification ability from tow aspects of validity and accuracy, constructs the whole weight allocation method of classifier and the evaluation function according to information entropy, and finally reduces the influence of subjective factors.
\end{abstract}

\section{Introduction}

Most faults of NC machine (in addition to sudden failures), the failures follow a gradual progression from scratch. In the course of failure, the state changes of NC machine tools do not actually have a definite boundary. In addition[1], the CNC machine itself is a complex system composed of many subsystems, and the uncertainty of the state of the fault diagnosis of CNC machine tools, as well as the uncertainty of the fault, is called fuzzy. Therefore, to a certain extent, the fault diagnosis process of CNC machine tools can be regarded as a model reasoning process[2], which is the theoretical basis of using fuzzy mathematics to solve the problem of numerical control fault diagnosis.

CNC machine tool fault complexity, concurrency characteristics, increase to establish the diagnosis model of fuzzy comprehensive evaluation is the fault model[3], factor sets the number of elements will have a greater impact on the final evaluation results. This is the weight allocation process, must satisfy all the evaluation factors, the sum of weights is 1 , this condition is decided. The method of solving such problems is to establish a multi-level fuzzy comprehensive evaluation model, and then to carry out high-level evaluation between categories[4]. The mathematical model of the two level fuzzy comprehensive evaluation can be recorded as:

$$
C=A \circ B=\left(\begin{array}{c}
A_{1} \circ R_{1} \\
A_{2} \circ R_{2} \\
\vdots \\
A_{n} \circ R_{n}
\end{array}\right)=A \circ\left(\begin{array}{c}
B_{1} \\
B_{2} \\
\vdots \\
B_{n}
\end{array}\right)
$$

Regardless of single level or multi-level fuzzy comprehensive evaluation model, each evaluation factor is not exactly the same in the concentration of factors. In order to reflect this difference, it is necessary to assign weights to each evaluation facto[5]r. Whether the weight is reasonable or not has a direct influence on the correctness of the final result, which is the most important part of the fuzzy comprehensive evaluation method. In this paper, the fuzzy synthetic evaluation model of CNC machine tool fault diagnosis based on information fusion is established by combining the information fusion technology with fuzzy comprehensive evaluation. 


\section{Establishment of Fuzzy Comprehensive Evaluation Model for Fault Diagnosis of NC Machine Tools}

Factor set U. In the establishment of the fuzzy comprehensive evaluation of classifier fusion model, evaluation object is no longer a number of features, but converted to the classification ability of each classifier to evaluate the following elements for each member classifier factor set[6]. If you assume that there are $\mathbf{J}$ member classifiers, the factor set is denoted as .

Judgement set $\mathbf{V}$. Each member of the classifier judges the type of the fault, and all the types of faults to be diagnosed constitute the set of judgments. If the number of fault types is , Set of judgement as .

Single factor judgement. Single factor judgment is defined as a single factor judgment, which is a fuzzy mapping from $U$ to $V, u_{i}=\mapsto\left(r_{i 1}, r_{i 2}, \cdots, r_{i m}\right)$.

The degree of membership given by classifier $u_{i}$ to fault type $v_{j}$ is $r_{i j}, r_{i j}$ needs to satisfy two conditions in equation 2 at the same time:

$$
\left\{\begin{array}{l}
0 \leq r_{i j} \leq 1,(i=1,2, \cdots, n ; j=1,2, \cdots, m) \\
\sum_{j=1}^{m} r_{i j}=1 .(i=1,2, \cdots, n)
\end{array}\right.
$$

All members of the classifier output are combined to form matrix R, called the judgment matrix. $(U, V, R)$ constitute the basic model of fuzzy comprehensive evaluation for fault diagnosis of CNC machine tools.

\section{Weight Allocation Method Based on Information Entropy}

Classification Capability Evaluation Function. Suppose there is a consists of three disjoint categories $C_{1}, C_{2}$ and $C_{3}$ of the decision space, a classifier 1 and 2 respectively for the same sample classification, if the expected output of a sample is $(1,0,0)$, the output of 1 search results for the $(0.95,0.05,0)$ classifier, classifier 2 output $(0.05,0.95,0)$. The output accuracy of the two classifiers is the same as the output of the classifier. Compared with the actual expected output, obviously, the diagnosis result of classifier 2 is different from the expected output, which shows that classifier 2 is classified. If the output of the classifier 1 is $(0.95,0.05,0)$, The output of the classifier 2 is $(0.60,0.40,0)$. Then according to the principle of maximum membership, two classifiers on the sample made the correct category judgment, but obviously, the result of classifier 2 is not as reliable as the result of classifier 1 , and the result can only be accepted in practice. A classifier with excellent classification ability should guarantee a certain diagnostic accuracy on the basis of sufficient diagnostic rate[7].

Weight Allocation. According to the previous analysis, the classifier's ability function is based on the complementary relation between the classifier classification ability[8], the evaluation function and the classifier classification ability:

$$
d_{j}=1-\overline{H_{e_{j}}}, j=1.2 . \cdots, J
$$

Then the weight of the classifier :

$$
\omega_{j}=\frac{d_{j}}{\sum_{j=1}^{J} d_{j}}
$$

Get weight vector $A=\left\{\omega_{1}, \omega_{2}, \cdots, \omega_{J}\right\}$.

Fusion Rule. After the weight vector $A$ and the judgment matrix $R$ are determined, the fuzzy comprehensive evaluation results are obtained: $B: B=A \circ R$. According to the method of weight 
determination, it is bound to satisfy: $\sum_{i=1}^{J} \omega_{i}=1$, and the classifier output is satisfied at the same time: $0 \leq y^{i}{ }_{j} \leq 1, \sum_{i=1}^{m} y_{j}^{i}=1$. It can be seen that the weight vector $A$ and the judgment matrix $R \quad$ are all normalized, then $\sum_{i=1}^{m} B_{i}=\sum_{j=1}^{J} \sum_{i=1}^{m} \omega_{i} y^{i}{ }_{j}=\sum_{j=1}^{J} \omega_{i} \cdot 1=1$.

So the output $B$ must satisfy the normalization requirements, namely $B=\left[b_{1}, b_{2}, \cdots, b_{m}\right]$, in the model, $b_{i}$ is the subordinate degree of the $i$ fault type. The fusion result $B=\left[b_{1}, b_{2}, \cdots, b_{m}\right]$ is a fuzzy vector, and the maximum membership principle is used to judge the fault type.

\section{Comprehensive Evaluation Based on Evaluation Matrix Classification Ability}

Steps are as follows:

1)First, the output of a fault type, the output of the test sample, the sample of the diagnosis conclusion and the expectation are calculated[9], and the evaluation function of the classifier diagnostic accuracy based on information entropy is calculated, The classification capability evaluation function $C_{i}, \forall i \in\{1,2, \cdots, m\}$ of the classifier $e_{j}, \forall j \in\{1,2, \cdots J\}$ for the fault type $H_{e_{j}}$ is obtained. The sample whose diagnosis is not in accordance with expectations does not have to be calculated at this step.

2)According to the number of samples, the evaluation function of classification capability $\overline{H_{e_{j}}}$ is obtained. In general, multiple fault types are tested with the same number of samples[10].

3) The misdiagnosis rate ${ }^{\sigma_{j}{ }^{C_{i}}}$ of statistical test samples, that is, the error rate of the classifier $e_{j}, \forall j \in\{1,2, \cdots J\}$ for the fault type $C_{i}, \forall i \in\{1,2, \cdots, m\}$.

Then defines the weight as $\omega_{y_{i}}^{{ }^{c_{i}}}=1-\sigma_{j}{ }^{{ }^{c_{i}}} \times \overline{H_{e_{j}}}$.

Let $\omega_{y_{i}}^{c_{i}}$ be classifier $e_{j}, \forall j \in\{1,2, \cdots J\}$ for fault type $C_{i}, \forall i \in\{1,2, \cdots, m\}$ weights. According to the above analysis, the fault classification ability evaluation matrix can be obtained $A^{\prime}$ :

$$
A^{\prime}=\left(\begin{array}{ccccc}
\omega_{y_{1}}{ }^{{ }_{1}} & \omega_{y_{2}}{ }^{c_{1}} & \cdots & \omega_{y_{J}}{ }^{c_{1}} \\
\omega_{y_{1}}{ }^{c_{2}} & \omega_{y_{2}}{ }^{c_{2}} & \cdots & \omega_{y_{J}}{ }^{c_{2}} \\
& \vdots & & \\
\omega_{y_{1}}{ }^{c_{m}} & \omega_{y_{2}}{ }^{c_{n}} & \cdots & \omega_{y_{J}}{ }^{c_{m}}
\end{array}\right)_{m \times J}
$$

Suppose there is a sample $X$, the output of the $J$ classifier is composed of the matrix to be evaluated $R$, remember to:

$$
R=\left(\begin{array}{ccc}
y_{1}{ }^{1} y_{1}{ }^{2} & \cdots & y_{1}{ }^{m} \\
y_{2}{ }^{1} y_{2}{ }^{2} & \cdots & y_{2}{ }^{m} \\
\vdots & \\
y_{J}{ }^{1} y_{J}{ }^{2} & \cdots & y_{J}^{m}
\end{array}\right)_{J \times m}
$$


According to the rules of fuzzy comprehensive evaluation:

$$
B_{m \times m}=A^{\prime} \bullet R=\left(b_{i j}\right)
$$

Among $b_{i j}=\omega_{y_{1}}{ }^{c_{1}} y_{1}{ }^{1}+\omega_{y_{2}}^{c_{1}} y_{2}{ }^{1}+\cdots+\omega_{y_{J}}{ }^{c_{m}} y_{J}{ }^{m}$.

After fusion, $\mathrm{B}$ is a $m \times m$ matrix, According to the rule of matrix multiplication, take the diagonal element, and obtain the vector composed of $m$ elements as the final result. In the classification capability evaluation matrix $A^{\prime}$, the weights are not normalized, so the output vectors are not normalized, but the fault types can still be judged according to the maximum membership principle.

\section{Fault Diagnosis Experiment of NC Machine Tool Based on Fuzzy Comprehensive Evaluation}

The BP model of the ball screw module is diagnosed, and the output of the BP model can be promoted as the possibility of various fault types, and the data obtained are normalized.

In the experiment, three classifiers, BP, RBF and SVM, are selected to classify and classify the randomly selected samples, and further fusion of the samples with diagnosis conflicts.

Table 1 Fusion Results of Rolling Bearing Modules

\begin{tabular}{|c|c|c|c|c|}
\hline $\begin{array}{c}\text { Sample serial } \\
\text { number }\end{array}$ & $\begin{array}{c}\text { Desired } \\
\text { output }\end{array}$ & \multicolumn{3}{|c|}{ Classifier fusion result } \\
\hline \multirow{2}{*}{1} & \multirow{2}{*}{$\mathrm{F} 1$} & 0.5732 & 0.4092 & 0.0040 \\
\hline & & 0.0077 & 0.0009 & 0.0059 \\
\hline \multirow{2}{*}{2} & \multirow{2}{*}{$\mathrm{F} 1$} & 0.5418 & 0.4365 & 0.0011 \\
\hline & & 0.0072 & 0.0080 & 0.0053 \\
\hline \multirow{2}{*}{3} & \multirow{2}{*}{$\mathrm{F} 1$} & 0.6768 & 0.2991 & 0.0072 \\
\hline & & 0.0101 & 0.0005 & 0.0062 \\
\hline \multirow{2}{*}{4} & \multirow{2}{*}{$\mathrm{F} 1$} & 0.5067 & 0.4063 & 0.0018 \\
\hline & & 0.0100 & 0.0135 & 0.0249 \\
\hline \multirow{2}{*}{5} & \multirow{2}{*}{$\mathrm{F} 2$} & 0.269 & 0.5660 & 0.0084 \\
\hline & & 0.1520 & 0.2305 & 0.0203 \\
\hline \multirow{2}{*}{6} & \multirow{2}{*}{$\mathrm{F} 2$} & 0.480 & 0.6982 & 0.0016 \\
\hline & & 0.2026 & 0.0233 & 0.0263 \\
\hline \multirow{2}{*}{7} & \multirow{2}{*}{$\mathrm{F} 2$} & 0.1839 & 0.4969 & 0.0186 \\
\hline & & 0.2347 & 0.0427 & 0.0231 \\
\hline \multirow{2}{*}{8} & \multirow{2}{*}{$\mathrm{F} 2$} & 0.145 & 0.5275 & 0.4291 \\
\hline & & 0.0030 & 0.0127 & 0.0132 \\
\hline \multirow{2}{*}{9} & \multirow{2}{*}{$\mathrm{F} 2$} & 0.206 & 0.3337 & 0.5796 \\
\hline & & 0.0346 & 0.0176 & 0.0140 \\
\hline \multirow{2}{*}{10} & \multirow{2}{*}{$\mathrm{F} 2$} & 0.708 & 0.5124 & 0.3918 \\
\hline & & 0.0038 & 0.0048 & 0.0164 \\
\hline \multirow{2}{*}{11} & \multirow{2}{*}{$\mathrm{F} 2$} & 0.173 & 0.0987 & 0.8457 \\
\hline & & 0.0136 & 0.0070 & 0.0177 \\
\hline \multirow{2}{*}{12} & \multirow{2}{*}{$\mathrm{F} 2$} & 0.146 & 0.0793 & 0.8638 \\
\hline & & 0.0213 & 0.0040 & 0.0169 \\
\hline \multirow{2}{*}{13} & & 0.3548 & 0.6222 & 0.0087 \\
\hline & $\mathrm{F} 2$ & 0.0037 & 0.0065 & 0.0041 \\
\hline 14 & F2 & 0.2105 & 0.7828 & 0.0011 \\
\hline 14 & $\mathrm{~F} 2$ & 0.0020 & 0.0028 & 0.0008 \\
\hline 15 & F2 & 0.4034 & 0.5357 & 0.0015 \\
\hline 15 & $\mathrm{~F} 2$ & 0.0121 & 0.0140 & 0.0332 \\
\hline & & 0.0065 & 0.3643 & 0.6113 \\
\hline 16 & F3 & 0.0079 & 0.0042 & 0.0058 \\
\hline & & 0.0161 & 0.4494 & 0.0045 \\
\hline 17 & $\mathrm{~F} 4$ & 0.4730 & 0.0379 & 0.0191 \\
\hline 18 & & 0.0093 & 0.4821 & 0.0151 \\
\hline 18 & $\mathrm{~F} 4$ & 0.0475 & 0.4346 & 0.0114 \\
\hline & & 0.0120 & 0.0205 & 0.0027 \\
\hline 19 & F5 & 0.5950 & 0.3549 & 0.0149 \\
\hline & & 0.0209 & 0.0531 & 0.0029 \\
\hline 20 & F5 & 0.4884 & 0.4140 & 0.0210 \\
\hline
\end{tabular}

\section{Conclusion}

The experiment saved to verify the feasibility of the fuzzy comprehensive evaluation model of fault diagnosis based on information fusion, but also verify the overall weighted average algorithm based 
on information entropy and effective evaluation method based on matrix improved the ability of classification.

Because does not have accurate mapping relationship between fault symptoms and fault reasons for this kind of complicated equipment, CNC machine tools, the given fault diagnosis method of fuzzy comprehensive evaluation, constructs a fuzzy comprehensive evaluation model for the diagnosis of CNC machine tools based on information fusion, in-depth study of the method of determining weight vector. In view of the uneven diagnosis ability of different fault types of classifier, the weight vector based on information entropy is improved into weighting matrix, which can evaluate the diagnosis ability of classifier more carefully.

\section{References}

[1] Meiling Qi. Application of intelligent fault diagnosis fusion technology in fault diagnosis of CNC machine tools[D]. Master Thesis of Dalian Jiaotong University, 2010.

[2] Chi Zhang. Typical fault analysis and diagnosis system design of NC machine tools. Master Thesis of Qingdao Technological University, 2010.

[3] Jin Du. Research on fault diagnosis of spindle servo system of NC machine tool[D]. Master Thesis of Nanjing University of Science and Technology,2010.

[4] Guoqiang Pu, Research on multisensor information fusion technology and mechanical fault diagnosis[J]. Guangxi Light Industry, 2010 (11) : 36-37

[5] Min Guo, Research on Application of multi-source information fusion in Fault Diagnosis Technology [J]. Computer and Digital Engineering, 2011 (3) : 1-4,71

[6] Jing Yuan,Zhengjia He,Yanyang Zi.Gear fault detection using cusromized multiwaveler lifting schemes[C],Mechanical Systems and Signal Processing,2010,24(5):1509-1528

[7] Jing Yuan, Zhengjia He, Yanyang Zi.Gear fault detection using customized multiwavelet lifting schemes[C],Mechanical Systems and Signal Processing,2010,24(5):1509-1528.

[8] Teysseer Satti, Ken Young, Samuel Grover. Detecting catastrophic failure events in large-scale milling machine[J], International Journal of Machine Tools and Manufacture,2009,49(14):1104-1113

[9] N. Saravanan, S. Cholairajan, K.I. Ramachandran. Vibrationg-based fault diagnosis of spur bevel gear box using fuzzy technique[J],Expert Systems with Applications, 2002,36(2):3119-3135

[10] HOU Zhao wen,ZHANG Zhou suo.Hybrid intelligent fault diagnosis based on granular computing[C].2009 IEEE International Conference on Granular Computing,Nanchang,China:219-224 